\title{
Relationships between the Prefrontal Cortex and the Basal Ganglia in the Rat: Physiology of the Corticosubthalamic Circuits
}

\author{
Nicolas Maurice, ${ }^{1}$ Jean-Michel Deniau, ${ }^{2}$ Jacques Glowinski, ${ }^{1}$ and Anne-Marie Thierry ${ }^{1}$ \\ ${ }^{1}$ Chaire de Neuropharmacologie, Institut National de la Santé et de la Recherche Médicale U 114, Collège de France, \\ 75231 Paris Cedex 05, France, and 2Université Pierre et Marie Curie, Département de Neurochimie-Neuroanatomie, \\ Institut des Neurosciences, Unité de Recherche Associée 1488, 75230 Paris Cedex 05, France
}

\begin{abstract}
The prelimbic-medial orbital areas (PL/MO) of the prefrontal cortex are connected to the medial part of the subthalamic nucleus (STN) through a direct projection and an indirect circuit that involves the core of the nucleus accumbens (NAcc) and the ventral pallidum (VP). In the present study, the influence of the PL/MO on the discharge of STN cells has been characterized. The major pattern of the responses observed after stimulation of PL/MO consisted of two excitatory peaks often separated by a brief inhibitory period. The early excitation was most likely to be caused by the activation of direct cortical inputs because its latency matches the conduction time of the prefrontal STN projections. The late excitation resulted from the activation of the indirect PL/MO-STN pathway that operates through a disinhibitory process. Indeed, the late excitation was no longer observed after acute blockade of the glutamatergic corticos-
\end{abstract}

triatal transmission by CNQX application into the NAcc. A similar effect was obtained after the blockade of the GABAergic striatopallidal transmission by bicuculline application into the VP. Finally, the brief inhibition that followed the early excitation was likely to result from the activation of a feedback inhibitory loop through VP because this inhibition was no longer observed after the blockade of STN inputs by CNQX application into the VP. This study further indicates the implication of STN in prefrontal basal ganglia circuits and underlines that in addition to a direct excitatory input, medial STN receives an indirect excitatory influence from PL/MO through an NAcc-VP-STN disinhibitory circuit.

Key words: basal ganglia circuits; prefrontal cortex; subthalamic nucleus; ventral striatum; nucleus accumbens; ventral pallidum; in vivo single unit recordings; rat
The subthalamic nucleus (STN) is a major component of the basal ganglia, and its critical role in the control of movement is well established. Pathological damage to the STN in humans or STN lesions in monkeys induce hemiballism (Whittier, 1947; Carpenter et al., 1950; Crossman, 1987). In 1-methyl-4-phenyl1,2,3,6-tetrahydropyridine-treated monkeys, an animal model of Parkinson's disease, abnormal activity of STN neurons has been observed, and lesions or high-frequency stimulation of the STN ameliorates akinesia and rigidity (Bergman et al., 1990; Benazzouz et al., 1993). High-frequency stimulation of the STN is now successfully applied to improve akinesia and rigidity in Parkinsonian patients (Limousin et al., 1995).

In current models of the basal ganglia circuitry, the striatum and the STN are the two major structures through which cortical signals are transmitted to the output structures of the basal ganglia, i.e., the substantia nigra pars reticulata (SNR) and the internal segment of the globus pallidus (GPi) (Alexander and Crutcher, 1990; Parent and Hazrati, 1995a,b). Indeed, both the striatum and the STN receive direct excitatory cortical inputs and send projections to the SNR and the GPi. Because the projection neurons of the striatum are GABAergic, whereas those of the

Received May 29, 1998; revised Aug. 26, 1998; accepted Aug. 31, 1998.

This work was supported by Institut National de la Santé et de la Recherche Médicale. Nicolas Maurice is recipient of a fellowship from the Ministère de l'Enseignement Supérieur et de la Recherche. We thank M. Saffroy and A. M. Godeheu for histological assistance and L. Darracq for his advice in the microdialysis technique.

Correspondence should be addressed to Dr. Anne-Marie Thierry, Chaire de Neuropharmacologie, Institut National de la Santé et de la Recherche Médicale U 114, Collège de France, 11 place Marcelin Berthelot, 75231 Paris Cedex 05, France. Copyright (ㄷ) 1998 Society for Neuroscience $\quad 0270-6474 / 98 / 189539-08 \$ 05.00 / 0$
STN are glutamatergic, these two structures exert opposing effects (inhibitory vs excitatory) on basal ganglia output neurons. In addition to the direct cortico-STN pathway, the cerebral cortex is connected to the STN through a multisynaptic circuit involving the striatum and the external segment of the globus pallidus (GPe). It has been proposed that through this indirect circuit the cerebral cortex activates STN via a disinhibitory process because the striatopallidal and the pallidosubthalamic pathways are GABAergic. However, the occurrence of such a disinhibitory process and the functional role of this indirect circuit is still debated and remains to be clarified.

In rat, the major cortical afferents to the STN originate from the motor and premotor areas (Afsharpour, 1985; Canteras et al., 1990). As shown more recently, the STN also receives projections from the prefrontal cortex, suggesting that it participates not only in sensorimotor but also in prefrontal circuits of the basal ganglia (Berendse and Groenewegen, 1991). In addition, we have recently shown that PL/MO areas of the prefrontal cortex may also influence the activity of STN cells through an indirect disinhibitory circuit that involves a restricted region of the ventral striatum, the core of nucleus accumbens (NAcc), and the ventral pallidum (VP) (Montaron et al., 1996; Maurice et al., 1997, 1998).

The present study was undertaken to determine the respective influence of the direct and indirect prefrontal cortex-subthalamic circuits on the discharge of STN neurons. The responses evoked by PL/MO stimulation on STN neurons were characterized, and the contribution of the direct cortico-STN and the indirect NAcc-VP-STN pathways on these responses was determined. For this purpose, we have reversibly blocked the synaptic trans- 
mission in the NAcc or in the VP and analyzed the responses of STN cells to PL/MO stimulation.

\section{MATERIALS AND METHODS}

Experiments were performed on 30 adult male Sprague Dawley rats (weight 275-300 gm; Charles River, St Aubin les Elbeuf, France). For all experimental procedures, rats were anesthetized with ketamine (100 $\mathrm{mg} / \mathrm{kg}$, i.p., supplemented by $50 \mathrm{mg} / \mathrm{kg}$, i.m.; Imalgène 500 , RhôneMérieux, France) and fixed in a conventional stereotaxic apparatus (Horsley Clarke Apparatus; Unimécanique, Epinay-sur-Seine, France). Body temperature was monitored and maintained at $37^{\circ} \mathrm{C}$ with an homeothermic warming blanket (Harvard Apparatus, Kent, UK).

Electrophysiological analysis. Single-unit activity was recorded extracellularly using glass micropipettes $(6-10 \mathrm{M} \Omega$ ) filled with a $0.6 \mathrm{~m}$ sodium chloride solution containing $4 \%$ Pontamine sky blue. Action potentials of single neurons were amplified with a World Precision Instruments DAM-5A differential preamplifier and displayed on a Tektronix memory oscilloscope. Spikes were separated from noise using a window discriminator and sampled on-line by a computer connected to a CED 1401 (Cambridge Electronic Design Ltd., Cambridge, UK) interface. Peristimulus time histograms were generated from 50-100 stimulation trials using $1 \mathrm{msec}$ bins and plotted on a Hewlett Packard plotter. The criterion used to establish the existence of an excitatory response was an increase $>50 \%$ in the number of spikes as compared with the prestimulus frequency for at least three consecutive bins. Medial prefrontal neurons were identified by antidromic activation after stimulation of the ipsilateral medial STN. In some experiments, STN neurons were identified by antidromic activation after stimulation of the ipsilateral medial SNR or the ipsilateral dorsal VP. Antidromic spikes were characterized by their fixed latency at threshold, their collision with spontaneous discharges within an appropriate time interval, and their ability to follow highfrequency stimulation.

Electrical stimulation of the PL/MO areas of prefrontal cortex, ipsilateral to the recording STN site, was performed through a co-axial stainless steel electrode (diameter, $400 \mu \mathrm{m}$; tip-barrel distance, $300 \mu \mathrm{m}$ ) positioned stereotaxically [anterior (A), 12.7; lateral (L), 0.6; height $(\mathrm{H})$, $5.5 \mathrm{~mm}$ from the interaural line] according to the atlas of Paxinos and Watson (1986). Electrical stimuli consisted of monopolar pulses of 0.6 msec width and $200-600 \mu \mathrm{A}$ intensity at a frequency of $1.4 \mathrm{~Hz}$. In some experiments, STN neurons were identified by their antidromic activation from the VP (A, 8.7; L, 2.5; H, 2.4) or from the SNR (A, 3.7; L, 1.8; H, 1.6).

At the end of each recording session, the tips of the stimulating electrodes were marked by an electrical deposit of iron (15 $\mu \mathrm{A}$ anodal, 20 $\mathrm{sec}$ ) and observed on histological sections after a ferriferrocyanide reaction. The tip of the recording electrode was marked by iontophoretic ejection of Pontamine sky blue ( $8 \mu \mathrm{A}$ cathodal, $20 \mathrm{~min}$ ), which allowed the determination of the position of the recording cells. Brains were removed and fixed in a $10 \%$ formalin solution, and the positions of electrodes were microscopically identified on serial frozen sections (100 $\mu \mathrm{m})$ stained with safranin.

Drug applications. Pharmacological blockade of the glutamatergic transmission in the NAcc and the VP or of the GABAergic transmission in the VP was performed by local application of CNQX and bicuculline, respectively. CNQX (500 $\mu \mathrm{M}$; Research Biochemicals, Natick, MA) and bicuculline (500 $\mu \mathrm{M}$; Sigma, St Louis, MO) were applied through a microdialysis probe (CMA 102; Microdialysis AB, Stockholm, Sweden; exposed length, $2 \mathrm{~mm})$. The microdialysis probes were positioned stereotaxically into the NAcc $(\mathrm{A}, 10.7 ; \mathrm{L}, 1.7 ; \mathrm{H}, 2.1)$ or the VP $(\mathrm{A}, 8.7 ; \mathrm{L}$, $2.5 ; \mathrm{H}, 1.4)$ according to the atlas of Paxinos and Watson (1986). At the beginning of each experiment, the probe was perfused with a Ringer's phosphate solution (in mM; $\mathrm{NaCl}, 120 ; \mathrm{KCl}, 4.8 ; \mathrm{CaCl}_{2}, 1.2 ; \mathrm{MgCl}_{2}, 1.2$; and $\left.\mathrm{NaH}_{2} \mathrm{PO}_{4}, 15.6\right)$ at a flow rate of $2 \mu \mathrm{l} / \mathrm{min}$. When an STN cell responding to $\mathrm{PL} / \mathrm{MO}$ stimulation was recorded, a peristimulus time histogram (60 stimuli) corresponding to the control situation was generated. The microdialysis pump was then permuted from the Ringer's solution to the antagonist solution. The activity of the cell was continuously recorded and, each fifth minute, its response to PL/MO stimulation was monitored and a peristimulus time histogram generated. The receptor antagonists were considered to have an effect when the responses evoked by PL/MO stimulation increased or decreased by at least $50 \%$. The drug was then washed out by perfusion with the Ringer's phosphate solution, and the same cell was recorded until the recovery of the control response. In cases in which more than one cell was tested in the same
A

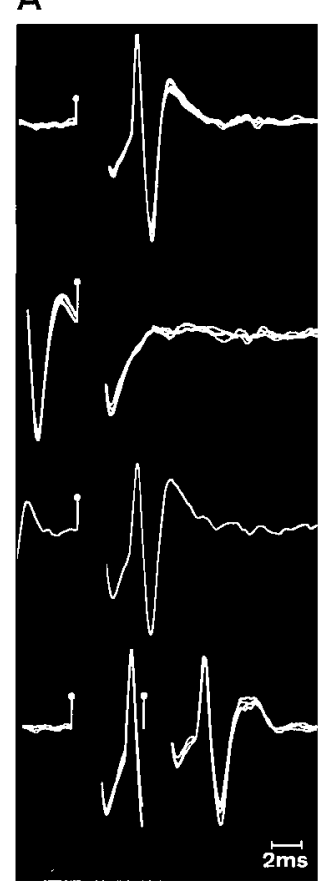

B

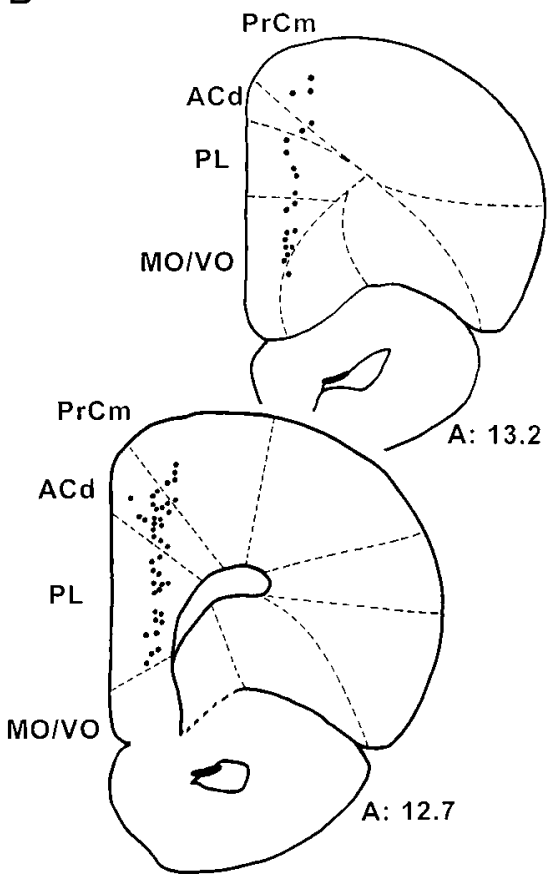

Figure 1. Localization of the cells in the prefrontal cortex antidromically driven by medial STN stimulation. $A$, Identification of the antidromic response elicited by STN stimulation in a prefrontal cortical cell. From top to bottom, Fixed latency $(3.4 \mathrm{msec})$, collision with spontaneous spikes, recovery of the antidromic response for a greater time interval between the spontaneous spikes and the stimulation, and ability to follow highfrequency stimulation $(250 \mathrm{~Hz})$. $B$, Localization of the antidromically driven cells in two frontal sections. Each dot represents a cell antidromically driven from the STN. Numbers indicate the distance in millimeters from the interaural line. $A C d$, Anterior cingulate area dorsal; $M O / V O$, medial orbital and ventral orbital areas; $P L$, prelimbic area; $P r C m$, medial precentral area.

animal, drug perfusions were separated by at least $2 \mathrm{hr}$ after the recovery of the control response in the preceding cell.

\section{RESULTS}

\section{Identification of medial prefrontal cortex neurons projecting to the subthalamic nucleus}

The neurons of the medial prefrontal cortex projecting to the STN were electrophysiologically identified using the antidromic activation method in three rats. Stimulation of the medial STN evoked an antidromic spike in $63(21.1 \%)$ of the 299 neurons recorded in the prefrontal cortex. The mean latency of the antidromic spikes was $4.5 \pm 0.2 \mathrm{msec}$ (range, $2.0-9.0 \mathrm{msec}$ ). As shown in Figure 1, the antidromically driven cells were mainly located within the layer $\mathrm{V}$ of the dorsal anterior cingulate and the prelimbic and the medial and ventral orbital areas of the prefrontal cortex.

\section{Effects of medial prefrontal cortex stimulation on the activity of subthalamic cells}

Electrical stimulation of the PL/MO areas evoked excitatory responses in 92 of the 141 STN cells recorded in 12 rats. As shown in Figure 2 and Table 1, different patterns of responses were observed. The major type of response (47 of the 92 responding cells) induced by PL/MO stimulation consisted in two excitatory peaks with short-latency $(7.6 \pm 0.2 \mathrm{msec})$ and long-latency $(21.4 \pm 0.5 \mathrm{msec})$ onsets. In most cases, the duration of the late 


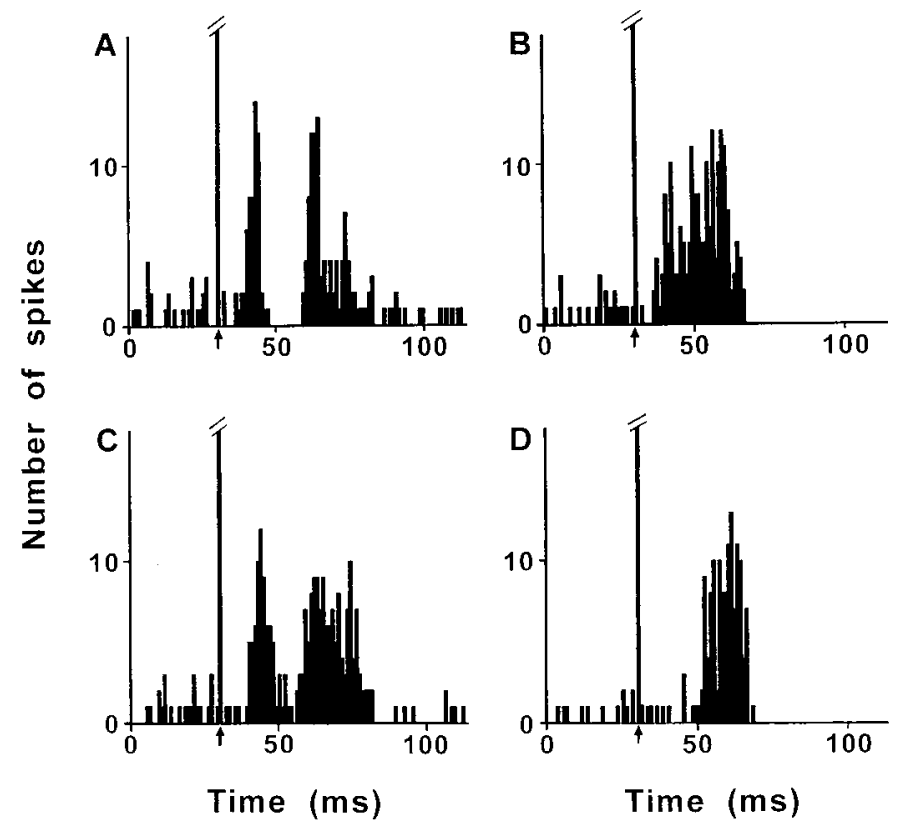

Figure 2. Patterns of responses evoked by PL/MO stimulation within STN cells. $A, C$, Two excitatory peaks with short- and long-latency onsets that are interrupted or uninterrupted by a brief inhibitory period; $B$, prolonged excitatory response with a short-latency onset; $D$, late excitatory response.

excitatory response was longer than the duration of the early excitatory peak. In 16 of these 47 cells, the two excitatory responses were separated by a short-duration inhibition [duration (D) $=6.7 \pm 0.4 \mathrm{msec}]$. In another population of cells $(20$ of the 92 responding cells), PL/MO stimulation induced a prolonged excitatory response with a short-latency $(8.2 \pm 0.5 \mathrm{msec})$ onset. Finally, a late $(21.2 \pm 0.6 \mathrm{msec})$ excitatory response was observed after PL/MO stimulation in the 25 remaining responding cells. In most cases (69 of the 92 responding cells), excitatory responses were followed by a long-lasting inhibition $(\mathrm{D}=155.2 \pm 10.9$ msec; range, 50-300 msec). As shown in Figure 3, the neurons that presented excitatory responses to PL/MO stimulation were located in the medial part of the STN, whereas the cells that did not respond were located more laterally. In the medial STN, the cells that presented distinct patterns of excitatory responses were observed in each animal and showed no obvious topographical distribution.

In some of these experiments ( 8 of the 12 rats) STN cells were identified by antidromic activation as projecting to either the medial SNR (four rats) or the VP (four rats). PL/MO stimulation induced excitatory responses in 25 of the 31 cells identified as projecting to the SNR (Fig. 4) and in all of the 13 cells identified as projecting to the VP. The different types of excitatory re- sponses described above were observed in both the cells projecting to the SNR or the VP.

\section{Effect of CNQX application into the NAcc on subthalamic excitatory responses evoked by PL/MO stimulation}

The effect of a blockade of the glutamatergic corticostriatal transmission by application of CNQX into the NAcc was examined in 10 STN cells that exhibited two excitatory peaks in response to PL/MO stimulation. CNQX application markedly decreased the late excitatory response in all the cells tested without significantly modifying the early excitatory response (Fig. 5). The maximal effect (52-96\% decrease) was observed 10-30 min after the beginning of CNQX application. In six of the cells held long enough, the recovery of the late excitatory response occurred 20-65 min after the cessation of CNQX application. Interestingly, although the two excitatory peaks were not separated by an inhibition in control conditions (9 of the 10 cells tested), under CNQX a short-duration inhibition [latency $(\mathrm{L})=17.8 \pm 1.2$ msec; D $=7.1 \pm 1.0 \mathrm{msec}$ ] appeared in seven cells (Fig. 5). Finally, in one cell in which the two excitatory peaks were separated by a short-lasting inhibition of $6 \mathrm{msec}$ in control conditions, this duration of the inhibition was increased to $16 \mathrm{msec}$ under CNQX.

\section{Effect of bicuculline application into the ventral pallidum on subthalamic excitatory responses evoked by PL/MO stimulation}

Bicuculline was applied into the VP to block the GABAergic transmission of the NAcc-VP pathway. The effect of bicuculline application on the excitatory responses evoked in STN by PL/MO stimulation was examined in eight cells. In five cells that exhibited two excitatory peaks in control conditions, bicuculline decreased the late excitatory response but not the early excitatory peak (Fig. 6 ). The maximal effect (50-98\% decrease) was observed 10-25 min after the beginning of bicuculline application, and recovery of the response occurred 30-80 min after the cessation of bicuculline application. While in control conditions, the two excitatory peaks were not interrupted by an inhibition, a short inhibitory period $(\mathrm{D}=8.7 \pm 1.1 \mathrm{msec})$ appeared during bicuculline application in three of these five cells (Fig. 6). In two cells that presented a prolonged excitatory response to PL/MO stimulation under control conditions, bicuculline induced decreased (77 and $84 \%$ ) excitatory responses $15 \mathrm{~min}$ after the beginning of bicuculline application, and the response recovery was observed $60 \mathrm{~min}$ after the cessation of bicuculline inf usion. Finally, in one cell that presented a late excitatory response to $\mathrm{PL} / \mathrm{MO}$ stimulation under control conditions, this excitation was decreased by $59 \% 8 \mathrm{~min}$ after the beginning of bicuculline application and recovered 25 min after the cessation of bicuculline application.

\section{Table 1. Characteristics of the excitatory responses evoked by PL/MO stimulation within STN cells}

\begin{tabular}{|c|c|c|c|c|c|}
\hline \multirow[b]{2}{*}{ Type of responses } & \multirow[b]{2}{*}{$\%$ of responding cells } & \multicolumn{2}{|c|}{ Early excitation } & \multicolumn{2}{|c|}{ Late excitation } \\
\hline & & $\mathrm{L}(\mathrm{msec})$ & $\mathrm{D}(\mathrm{msec})$ & $\mathrm{L}(\mathrm{msec})$ & $\mathrm{D}(\mathrm{msec})$ \\
\hline Two excitations $^{a}$ & $51.1 \%(n=47)$ & $7.6 \pm 0.2$ & $6.1 \pm 0.3$ & $21.4 \pm 0.5$ & $14.3 \pm 0.6$ \\
\hline One prolonged excitation & $21.7 \%(n=20)$ & $8.2 \pm 0.5$ & $23.8 \pm 0.9$ & & \\
\hline One late excitation & $27.2 \%(n=25)$ & & & $21.2 \pm 0.6$ & $12.2 \pm 0.8$ \\
\hline
\end{tabular}

${ }^{a}$ In 16 of these 47 cells, a short inhibition $(\mathrm{L}=15.3 \pm 0.6 \mathrm{msec} ; \mathrm{D}=6.7 \pm 0.4 \mathrm{msec})$ separated the two excitatory peaks; $n$, number of cells. 


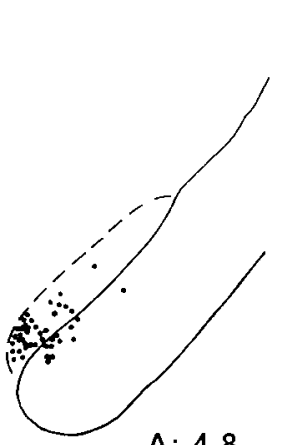

A: 4.8

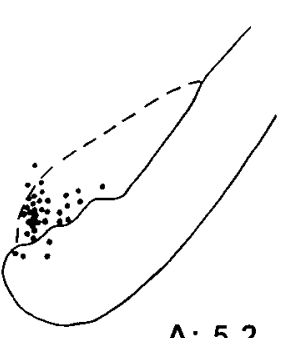

A: 5.2

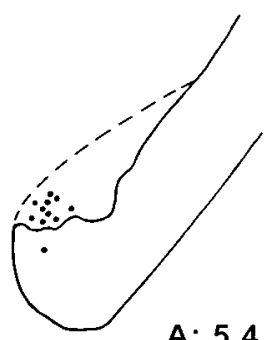

A: 5.4
Not responding cells

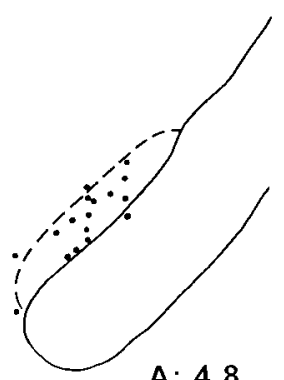

A: 4.8

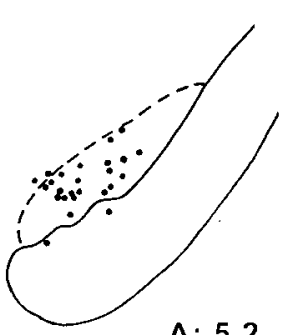

A: 5.2

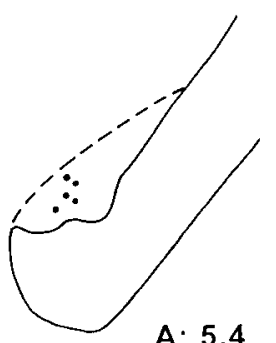

A: 5.4
Figure 3. Localization of STN cells that presented excitatory responses to PL/MO stimulation. Note that responding cells are located in the medial part of the STN, whereas more laterally located cells do not respond to PL/MO stimulation (Not responding cells). Each dot represents a tested cell. Numbers indicate the distance in millimeters from the interaural line.
Effect of CNQX application into the ventral pallidum on subthalamic excitatory responses evoked by PL/MO stimulation

Because the STN and the VP are reciprocally connected, CNQX was applied into the VP to block the glutamatergic input from the STN and to investigate the possible influence of this circuit on the $\mathrm{PL} / \mathrm{MO}$-evoked responses. The effect of CNQX application into the $\mathrm{VP}$ on the excitatory responses evoked by $\mathrm{PL} / \mathrm{MO}$ stimulation was examined in nine STN cells. In eight cells that produced two excitatory peaks in control conditions, the application of CNQX induced an increase of the late excitatory response, a maximal effect (66-250\%) occurring 20-30 min after the beginning of CNQX infusion (Fig. 7). The duration of this late excitation (D = $14.5 \pm 1.1 \mathrm{msec}$, control conditions) was significantly increased $(\mathrm{D}=26.6 \pm 2.0 \mathrm{msec} ; p<0.001)$ under $\mathrm{CNQX}$ application without a significant change of the latency (control, $\mathrm{L}=23.1 \pm$ $1.0 \mathrm{msec}$; CNQX, $\mathrm{L}=21.4 \pm 1.2 \mathrm{msec}$ ). In three cells, a slight $(45 \%)$ increase in the early excitatory peak was also observed. In two cells, in which the early and late excitatory responses were separated by an inhibition in control conditions, this inhibition disappeared under CNQX (Fig. 7). In five cells that were held long enough, the recovery of responses was observed 45-75 min after the cessation of CNQX perfusion. Finally, in one cell responding to $\mathrm{PL} / \mathrm{MO}$ stimulation by a late excitatory response $(\mathrm{L}=22 \mathrm{msec} ; \mathrm{D}=11 \mathrm{msec})$ under control conditions, the response was increased by $220 \%(\mathrm{~L}=18 \mathrm{msec} ; \mathrm{D}=35 \mathrm{msec}) 20$ min after the beginning of CNQX application.

\section{DISCUSSION}

The present study was undertaken to determine the functional characteristics of the PL/MO-STN circuits in the rat. The data show that $\mathrm{PL} / \mathrm{MO}$ stimulation induced excitatory responses in the
Figure 4. Excitatory responses evoked by $\mathrm{PL}$ area stimulation in an STN cell antidromically identified as projecting to the medial SNR. Left, Identification of the antidromic response. From top to bottom, Fixed latency $(3.3 \mathrm{msec})$, collision with spontaneous spikes, recovery of the antidromic response for a greater time interval between the spontaneous spike and the stimulation, and ability to follow high-frequency stimulation $(200 \mathrm{~Hz})$. Right, Top, Excitatory response evoked by PL stimulation in the same STN cell (poststimulus time histogram, 50 superimposed sweeps); bottom, interspike interval histogram (500 intervals) illustrating the spontaneous activity of the cell (left) and the experimental design (right). $P L$, Prelimbic area; $S N R$, substantia nigra pars reticulata; STN, subthalamic nucleus.
SNR stimulation

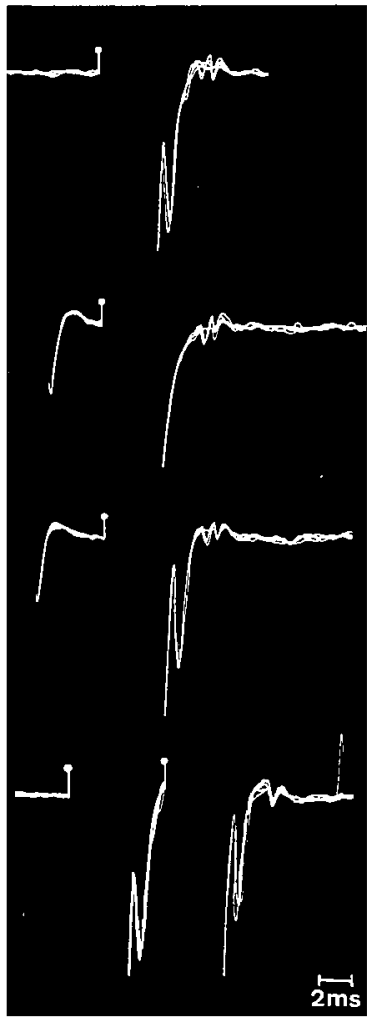

PL stimulation
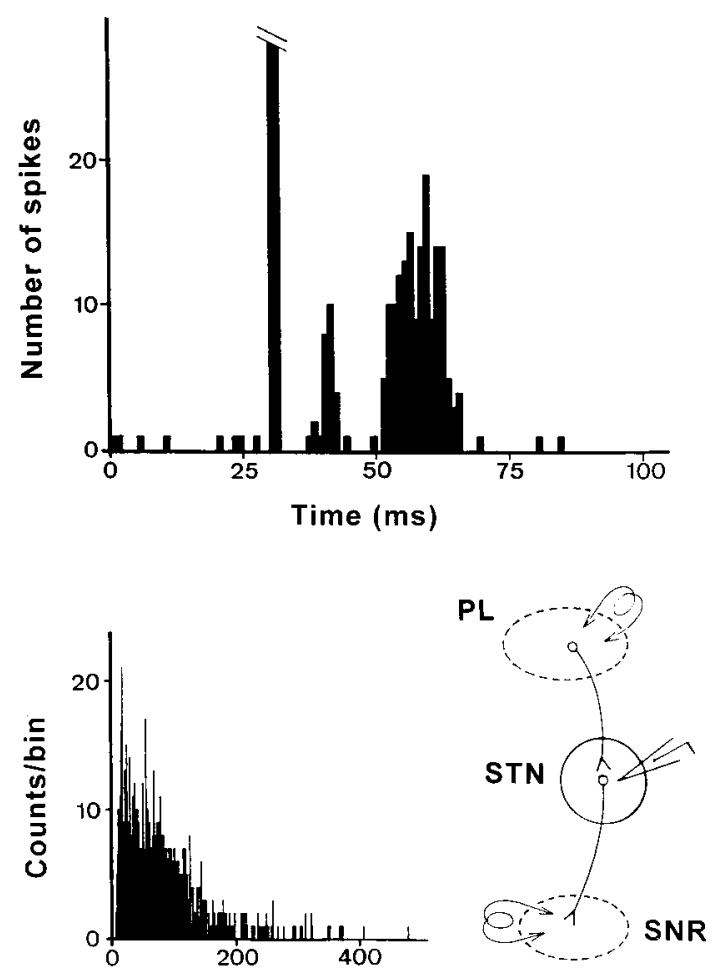

Interspike Interval (ms) 

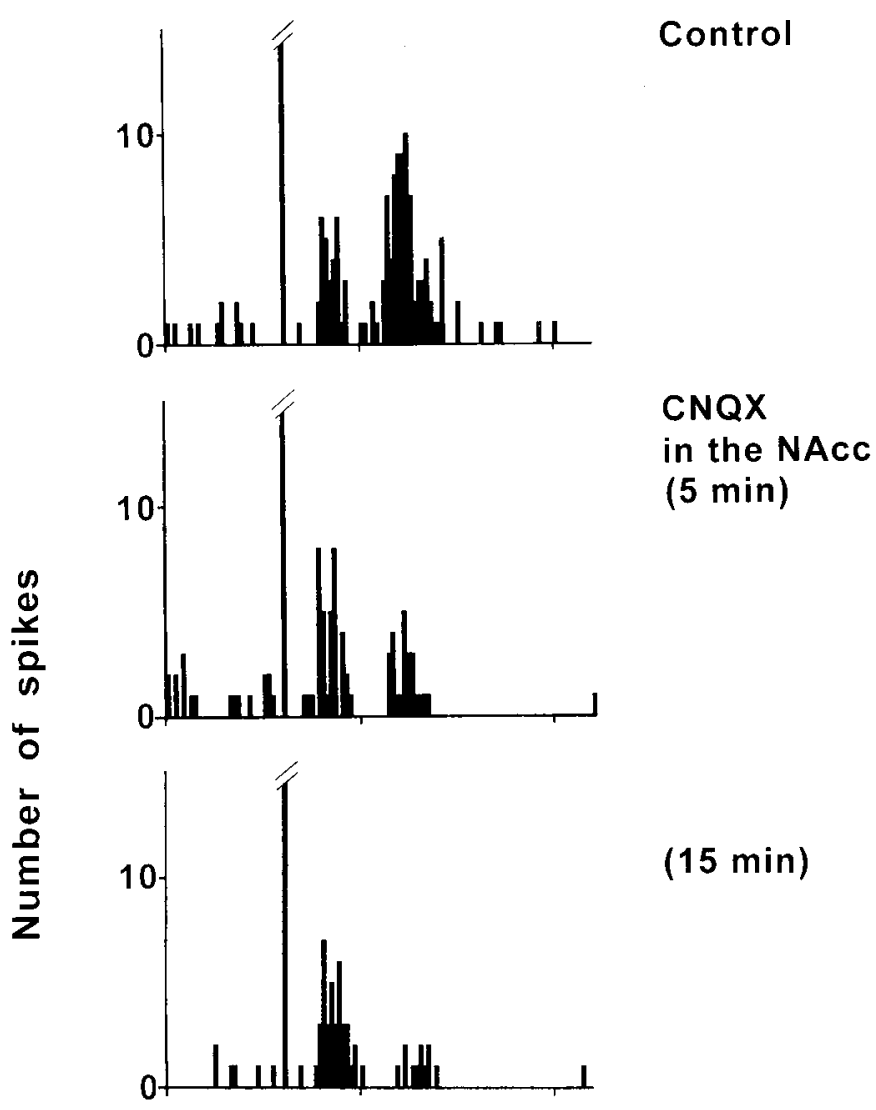

$(15 \mathrm{~min})$

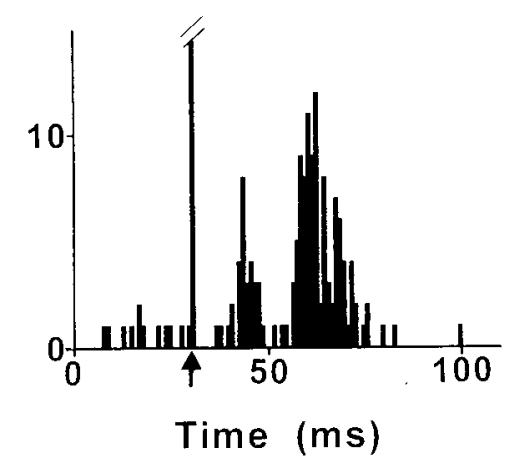

Recovery

Figure 5. Effect of CNQX application into the NAcc on the response evoked by PL/MO stimulation in an STN cell. From top to bottom, The response exhibited two excitatory peaks in control conditions, CNQX application into the NAcc did not significantly modify the early peak but markedly decreased the late excitatory response, the maximal effect was observed $15 \mathrm{~min}$ after the beginning of CNQX application, and the recovery of the late excitatory response occurred 25 min after the cessation of CNQX application. Note that although the two excitatory peaks were not separated by an inhibition in control conditions (Control, Recovery), during CNQX application a short inhibitory period was observed. Each poststimulus time histogram represents 60 superimposed sweeps. Arrow indicates the stimulation artifact.

medial part of the STN, which mainly consisted of two excitatory peaks separated in some cases by a brief inhibitory period. The early response is likely caused by the activation of the direct prefrontal cortex-STN projection, whereas the late response involves the indirect cortico-NAcc-VP-STN pathway. Indeed, application of CNQX into the NAcc or bicuculline into the VP blocked the late but not the early excitatory peak. In addition, the present data indicate that the VP is also involved in an inhibitory feedback control of the STN through the reciprocal circuit.

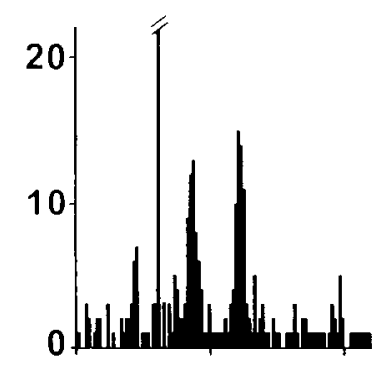

Control

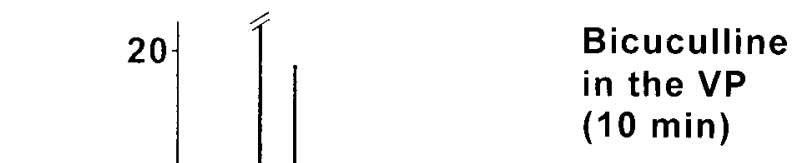

$(20 \mathrm{~min})$

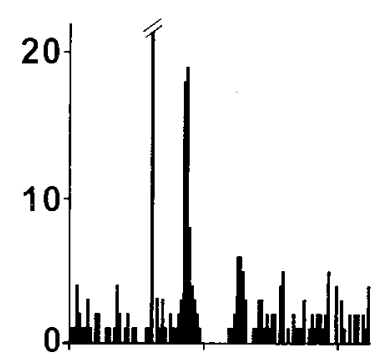

Recovery

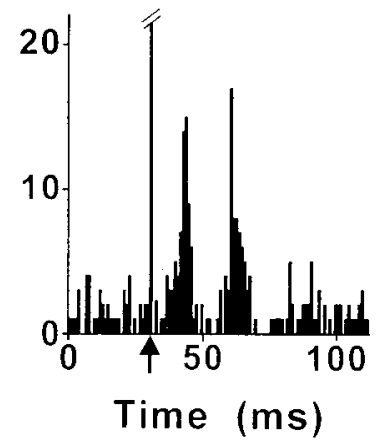

Figure 6. Effect of bicuculline application into the VP on the response evoked by PL/MO stimulation in an STN cell. From top to bottom, The response exhibited two excitatory peaks in control conditions, bicuculline application into the VP decreased the late excitatory response without modifying the early excitatory peak, the maximal effect was observed 20 min after the beginning of bicuculline application, and the recovery of the late excitatory response occurred $35 \mathrm{~min}$ after the cessation of bicuculline application. Note that, although the two excitatory peaks were not separated by an inhibitory period in the control conditions (control, recovery), during the infusion of bicuculline a short-duration inhibition appeared. Each poststimulus time histogram represents 60 superimposed sweeps. Arrow indicates the artifact of stimulation.

\section{Origin of the early excitatory response}

Converging evidence indicates that cortico-STN projections use glutamate as neurotransmitter. Cortical terminals are enriched in glutamate and form asymmetrical synaptic contacts with the dendrites of subthalamic neurons (Bevan et al., 1995). The synaptic effects of the cortico-STN pathway are well established for STN inputs from the sensorimotor cortex. Stimulation of the sensorimotor cortex in the rat evokes short-latency monosynaptic excitatory responses in STN cells that are blocked by local application 


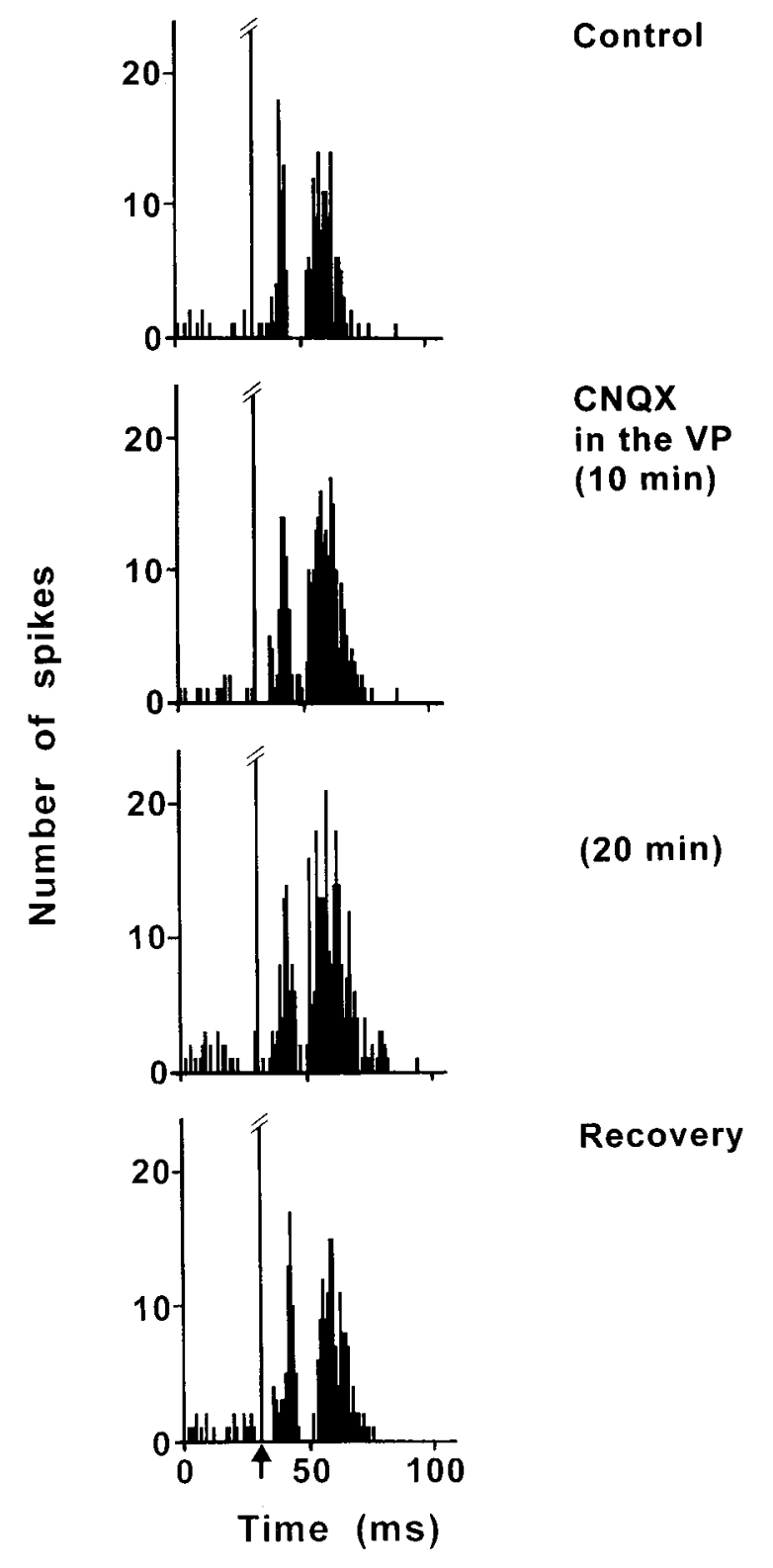

Figure 7. Effect of CNQX application into the VP on the response evoked by PL/MO stimulation in an STN cell. From top to bottom, The response exhibited two excitatory peaks separated by a brief inhibitory period in the control conditions, CNQX application into the VP induced an enhancement of the late excitatory response and a disappearance of the inhibitory period, the maximal effect was observed $20 \mathrm{~min}$ after the beginning of CNQX application, and the recovery of the responses occurred $50 \mathrm{~min}$ after the cessation of CNQX application. Each poststimulus time histogram represents 60 superimposed sweeps. Arrow indicates the artifact of stimulation.

of glutamatergic antagonists (Kitai and Deniau, 1981; RouzaireDubois and Scarnati, 1987; Ryan and Clark, 1991; Fujimoto and Kita, 1993). Early excitatory responses evoked by PL/MO stimulation in the STN are also likely to be monosynaptic. Indeed, the latency of such early responses was found to be in the range of the conduction time of prefrontal-STN pathway determined by antidromic activation. Moreover, in agreement with the topographical organization of prefrontal-STN projections cells that responded to PL/MO stimulation were located in the medial STN (Berendse and Groenewegen, 1991). It has been previously re-

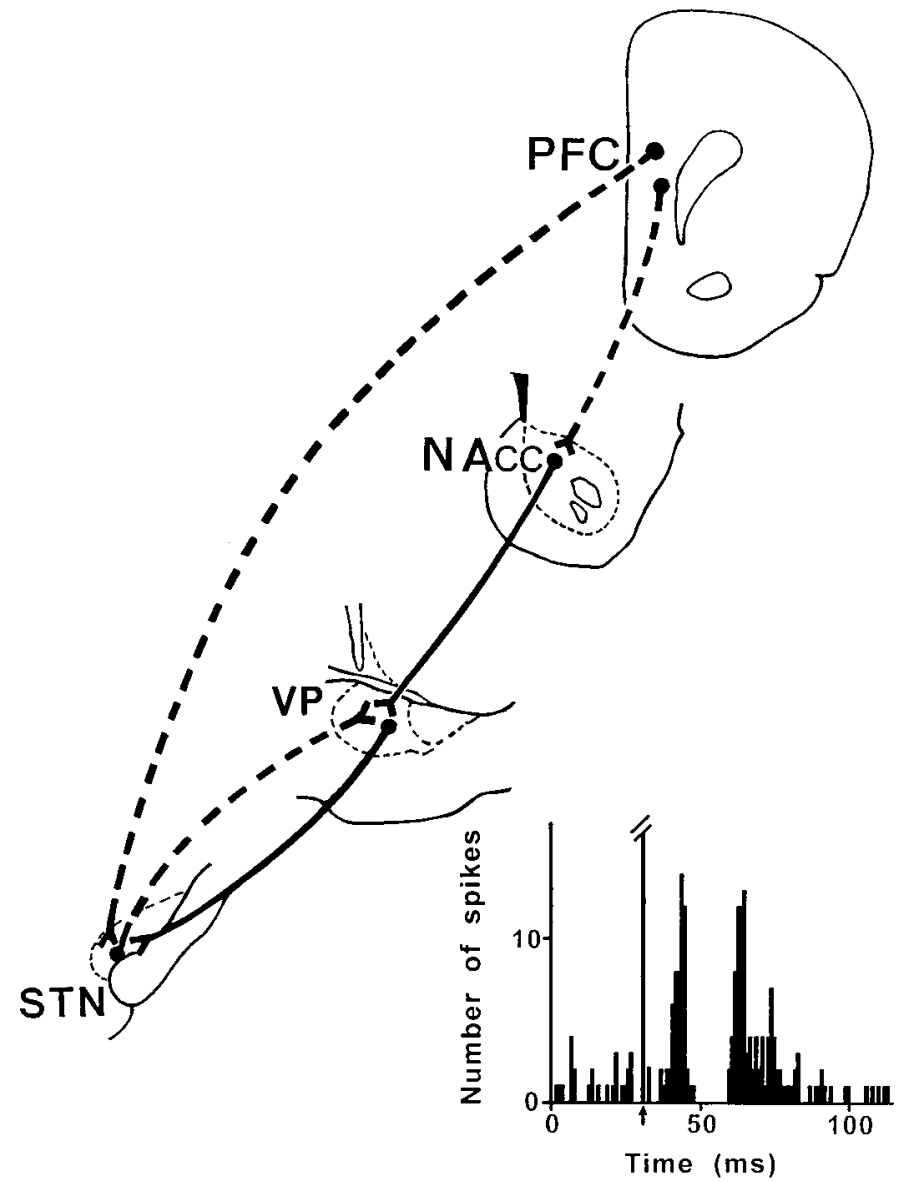

Figure 8. Schematic representation of the pathways involved in the STN responses to prefrontal cortical stimulation. Broken and solid lines represent glutamatergic and GABAergic pathways, respectively. Bottom, Example of a complex response evoked in an STN cell by prefrontal cortex stimulation. The early excitatory peak is likely to be caused by the activation of a direct cortico-STN projection. The brief inhibition that follows the early excitatory peak results from the activation of a feedback inhibitory circuit through the VP. The late excitatory response involves an indirect cortico-NAcc-VP-STN pathway that operates via a disinhibitory process. NAcc, Nucleus accumbens; $P F C$, prefrontal cortex; $S T N$, subthalamic nucleus; $V P$, ventral pallidum.

ported that stimulation of the prelimbic cortex evoked a late, but not an early excitation of STN cells (Ryan and Clark, 1991). This could be explained by the localization of the recording sites because, in our study, cells that responded to PL/MO stimulation were found in the medial STN and not more laterally.

\section{Origin of the late excitatory response}

Previous findings have indicated that electrical stimulation of the sensorimotor cortex evokes two excitatory peaks within STN neurons, often interrupted by a brief inhibition (Ryan and Clark, 1991; Fujimoto and Kita, 1993). However, the origin of the second excitatory peak remained unclear. The involvement of the indirect striato-pallido-STN circuit has been questioned from data obtained in lesioned rats. Indeed, after excitotoxic lesions of the striatum or the GPe, the pattern of excitatory responses to cortical stimulation was almost the same as in intact rats, and an enhancement of the late excitatory component has also been reported (Ryan and Clark, 1992; Fujimoto and Kita, 1993). Therefore, the second excitatory peak has been considered as the late component of a single broad excitatory response interrupted 
by a brief inhibition (Fujimoto and Kita, 1993). Several explanations for the prolonged excitations have been proposed: activation of NMDA receptors, amplification of small excitatory synaptic inputs by the electrical membrane characteristics of STN cells, and the spread of excitations within the STN through local axon collaterals (for review, see Kita, 1994).

In contrast to the lesion experiments, the present study shows that the late excitatory response induced by PL/MO stimulation in the STN was markedly reduced after acute blockade of the corticostriatal neurotransmission by local application of CNQX into the NAcc. A similar effect was observed after blockade of the striatopallidal neurotransmission by local application of bicuculline into the VP. In both cases, the duration of the application of receptor antagonists required to obtain a maximal effect showed some variability. This might be related to the diffusion time necessary for the antagonist to block the synaptic transmission in the whole NAcc or VP territory involved in the PL/MO-STN circuit. Interestingly, the early excitatory peak was not affected by local applications of CNQX or bicuculline. Thus, it can be concluded that the early and late excitatory responses that are evoked in STN cells by PL/MO stimulation result from the activation of two distinct pathways, the direct cortico-STN pathway and the indirect striato-pallido-subthalamic circuit, respectively. The apparent discrepancy between the data reported after excitotoxic lesions and the present study using acute blockade of the synaptic transmission may be caused by a subsequent reorganization of synaptic inputs to STN cells and/or modification of their excitability after lesions.

Altogether, the present data and those of our previous studies (Maurice et al., 1997, 1998) indicate that late excitatory responses involving the indirect cortico-striato-pallido-subthalamic circuit result from a disinhibitory process. Indeed, stimulation of $\mathrm{PL} / \mathrm{MO}$ areas induces an activation of NAcc core neurons that send an inhibitory input on the VP-STN cells. In addition, VP stimulation elicits an inhibition of STN cells located in the medial part of this nucleus. Thus, phasic activation of the striatopallidal pathway leads to an inhibition of the tonically active inhibitory pallidosubthalamic neurons, resulting in a disinhibition of STN. Also in favor of a disinhibitory process, the latency of the late excitatory responses is compatible with the conduction time of neurons within the indirect cortico-striato-pallido-subthalamic circuit (Montaron et al., 1996; Maurice et al., 1997). The efficacy of this disinhibitory process could partly be caused by the high input resistance and the resting membrane potential of the STN cells, which is close to the threshold for a spike (Nakanishi et al., 1987).

\section{Origin of the short-duration inhibitory response}

STN and pallidum are two tightly interconnected structures (Kita et al., 1983; Robledo and Féger, 1990; Kita and Kitai, 1991; Ryan and Clark, 1992; Shink et al., 1996; Maurice et al., 1997, 1998). A topographical organization of their reciprocal connections has been described: GPe and VP receive inputs from different parts of the STN and project back to the STN region from which they receive inputs (Groenewegen and Berendse, 1990; Joel and Weiner, 1997).

Because the STN projections to the pallidum are excitatory, it has been proposed that the activation of the STN cells projecting to pallidum by the direct cortical inputs leads to an activation of the GABAergic pallidosubthalamic neurons that result in a feedback inhibition of the STN (Ryan and Clark, 1991; Fujimoto and Kita, 1993). Accordingly, the present data indicate that the brief inhibitory period that follows the early excitatory peak induced by $\mathrm{PL} / \mathrm{MO}$ stimulation in STN cells is likely to be caused by the activation of a feedback inhibitory circuit through VP. Indeed, $\mathrm{PL} / \mathrm{MO}$ stimulation induced early excitatory responses in the STN cells identified as projecting to the VP. In addition, the brief inhibitory period that follows the early excitatory peak was no longer observed after blockade of the glutamatergic STN-VP transmission by CNQX application into VP.

\section{Functional considerations}

In current working models of the basal ganglia (Alexander and Crutcher, 1990; Parent and Hazrati, 1995b; Joel and Weiner, 1997), STN is mainly viewed as a relay nucleus of the transstriatal indirect pathway and is also considered as an input structure because of its direct cortical afferents. The STN, the only glutamatergic structure within the basal ganglia, provides a major excitatory drive on output structures that counteracts the inhibitory influence exerted by the direct GABAergic striatopallidal and striatonigral pathways. It has been assumed that a functional imbalance between the direct striatal projections and the transSTN circuits underlies the dyskinesias observed in parkinsonism, chorea, and ballism (Albin et al., 1989; Wichmann and DeLong, 1996; Obeso et al., 1997).

Our investigation on the functional relationships between the prefrontal cortex and the STN indicates that the cortical excitatory influence on STN cells involves not only the direct corticoSTN pathway but also the indirect cortico-striato-pallidosubthalamic circuit that operates through a disinhibitory process (Fig. 8). Although these two pathways can converge on the same STN cells, they might subserve different functions. Indeed, they have different conduction times and mainly originate from distinct cortical layers (layer $\mathrm{V}$ in the case of the direct pathway and superficial layers in the case of the indirect transstriatal circuit), suggesting that they are activated under different conditions (Montaron et al., 1996; present study). Finally, the present data indicate that, by its direct excitatory input on STN-VP projecting neurons, the prefrontal cortex can activate a feedback inhibitory loop and subsequently exert an inhibitory control on the STN (Fig. 8). Thus, the VP participates in two intrinsic basal ganglia circuits that exert opposite influences on the discharge of STN cells. It might, therefore, be important to account for this dual and opposing role of the pallidum (VP and GPe) in the pathophysiological models of the basal ganglia.

In conclusion, the STN, which is primarily implicated in the control of motor functions, also participates in the limbic and cognitive functions of basal ganglia as suggested in recent behavioral studies (Baunez et al., 1995). In agreement with the concept of a parallel architecture of the corticobasal ganglia circuits, information arising from prefrontal and sensorimotor cortical areas are segregated within the STN. It remains to determine the specific role of the medial STN in the control of limbic and cognitive functions of the prefrontal cortex.

\section{REFERENCES}

Afsharpour S (1985) Topographical projections of the cerebral cortex to the subthalamic nucleus. J Comp Neurol 236:14-28.

Albin RL, Young AB, Penney JB (1989) The functional anatomy of basal ganglia disorders. Trends Neurosci 12:366-375.

Alexander GE, Crutcher MD (1990) Functional architecture of basal ganglia circuits: neural substrates of parallel processing. Trends Neurosci 13:266-271.

Baunez C, Nieoullon A, Amalric M (1995) In a rat model of parkinsonism, lesions of the subthalamic nucleus reverse increases of reaction 
time but induce a dramatic premature responding deficit. J Neurosci 15:6531-6541.

Benazzouz A, Gross C, Féger J, Boraud T, Bioulac B (1993) Reversal of rigidity and improvement in motor performance by subthalamic highfrequency stimulation in MPTP-treated monkeys. Eur J Neurosci 5:382-389.

Berendse HW, Groenewegen HJ (1991) The connections of the medial part of the subthalamic nucleus in the rat: evidence for a parallel organization. In: The basal ganglia III. (Bernardi G, Carpenter MB, Di Chiara G, Morelli M, Stanzione P, eds), pp 89-98. New York: Plenum.

Bergman H, Wichmann T, DeLong MR (1990) Reversal of experimental parkinsonism by lesions of the subthalamic nucleus. Science 249:1436-1438.

Bevan MD, Francis CM, Bolam JP (1995) The glutamate-enriched cortical and thalamic input to neurons in the subthalamic nucleus of the rat: convergence with GABA-positive terminals. J Comp Neurol 361:491-511.

Canteras NS, Shammah-Lagnado SJ, Silva BA, Ricardo JA (1990) Afferent connections of the subthalamic nucleus: a combined retrograde and anterograde horseradish peroxidase study in the rat. Brain Res 513:43-59.

Carpenter MB, Whittier JR, Mettler FA (1950) Analysis of choreoid hyperkinesia in the rhesus monkey: surgical and pharmacological analysis of hyperkinesia resulting from lesions in the subthalamic nucleus of luys. J Comp Neurol 92:293-332.

Crossman AR (1987) Primate models of dyskinesia: the experimental approach to the study of basal ganglia-related involuntary movement disorders. Neuroscience 21:1-40.

Fujimoto K, Kita H (1993) Response characteristics of subthalamic neurons to the stimulation of the sensorimotor cortex in the rat. Brain Res 609:185-192.

Groenewegen HJ, Berendse HW (1990) Connections of the subthalamic nucleus with ventral striatopallidal parts of the basal ganglia in the rat. J Comp Neurol 294:607-622.

Joel D, Weiner I (1997) The connections of the primate subthalamic nucleus: indirect pathways and the open-interconnected scheme of basal ganglia-thalamocortical circuitry. Brain Res Rev 23:62-78.

Kita H (1994) Physiology of two disynaptic pathways from the sensorimotor cortex to the basal ganglia output nuclei. In: The basal ganglia IV (Percheron G, McKenzie JS, Féger J, eds), pp 263-276. New York: Plenum.

Kita H, Kitai ST (1991) Intracellular study of rat globus pallidus neurons: membrane properties and responses to neostriatal, subthalamic and nigral stimulation. Brain Res 564:296-305.

Kita H, Chang HT, Kitai ST (1983) Pallidal inputs to subthalamus: intracellular analysis. Brain Res 264:255-265.

Kitai ST, Deniau JM (1981) Cortical inputs to the subthalamus: intracellular analysis. Brain Res 214:411-415.

Limousin P, Pollak P, Benazzouz A, Hoffmann D, Le Bas JF, Broussolle
E, Perret JE, Benabid AL (1995) Effect on parkinsonian signs and symptoms of bilateral subthalamic nucleus stimulation. Lancet 345:91-95.

Maurice N, Deniau JM, Ménétrey A, Glowinski J, Thierry AM (1997) Position of the ventral pallidum in the rat prefrontal cortex-basal ganglia circuit. Neuroscience 80:523-534.

Maurice N, Deniau JM, Ménétrey A, Glowinski J, Thierry AM (1998) Prefrontal cortex-basal ganglia circuits in the rat: Involvement of ventral pallidum and subthalamic nucleus. Synapse 29:363-370.

Montaron MF, Deniau JM, Ménétrey A, Glowinski J, Thierry AM (1996) Prefrontal cortex inputs of the nucleus accumbens-nigrothalamic circuit. Neuroscience 71:371-382.

Nakanishi H, Kita H, Kitai ST (1987) Electrical membrane properties of rat subthalamic neurons in an in vitro slice preparation. Brain Res 437:35-44.

Obeso JA, Rodriguez MC, DeLong MR (1997) Basal ganglia pathophysiology: a critical review. In: The basal ganglia and new surgical approaches for Parkinson's disease (Obeso JA, DeLong MR, Ohye C, Marsden CD, eds), pp 3-18. Philadelphia: Lippincott-Raven.

Parent A, Hazrati LN (1995a) Functional anatomy of the basal ganglia. I. The cortico-basal ganglia-thalamo-cortical loop. Brain Res Rev 20:91-127.

Parent A, Hazrati LN (1995b) Functional anatomy of the basal ganglia. II. The place of subthalamic nucleus and external pallidum in basal ganglia circuitry. Brain Res Rev 20:128-154.

Paxinos G, Watson C (1986) The rat brain in stereotaxic coordinates, Ed 2. New York: Academic.

Robledo P, Féger J (1990) Excitatory influence of rat subthalamic nucleus to substantia nigra pars reticulata and the pallidal complex: electrophysiological data. Brain Res 518:47-54.

Rouzaire-Dubois B, Scarnati E (1987) Pharmacological study of the cortical-induced excitation of subthalamic nucleus neurons in the rat: evidence for amino acids as putative neurotransmitters. Neuroscience 21:429-440.

Ryan LJ, Clark KB (1991) The role of the subthalamic nucleus in the response of globus pallidus neurons to stimulation of the prelimbic and agranular frontal cortices in rats. Exp Brain Res 86:641-651.

Ryan LJ, Clark KB (1992) Alteration of neuronal responses in the subthalamic nucleus following globus pallidus and neostriatal lesions in rats. Brain Res Bull 29:319-327.

Shink E, Bevan MD, Bolam JP, Smith Y (1996) The subthalamic nucleus and the external pallidum: two tightly interconnected structures that control the output of the basal ganglia in the monkey. Neuroscience 73:335-357.

Whittier JR (1947) Ballism and the subthalamic nucleus (nucleus hypothalamicus; corpus Luysii). Arch Neurol Psychiatry 58:672-692.

Wichmann T, DeLong MR (1996) Functional and pathophysiological models of the basal ganglia. Curr Opin Neurobiol 6:751-758. 